\title{
Is angiography still the best method to stratify stroke risk in symptomatic atherosclerotic carotid plaque?
}

\author{
Gustavo W. Kuster ${ }^{1^{*}}$, A. J. Da Rocha ${ }^{2}$, A. S. F. Silva ${ }^{2}$, R. A. Valiente ${ }^{3}$, M. S. G. Rocha ${ }^{3}$, \\ A. R. Massaro ${ }^{4}$ \\ ${ }^{1}$ Division of Neurology, São Paulo University, São Paulo, Brazil; ${ }^{*}$ Corresponding Author: gwkuster@hotmail.com \\ ${ }^{2}$ Department of Radiology and Pathology, Fleury Institute, São Paulo, Brazil \\ ${ }^{3}$ Division of Neurology, Santa Marcelina Hospital, São Paulo, Brazil \\ ${ }^{4}$ Sao Paulo University, São Paulo, Brazil
}

Received 20 June 2013; revised 18 July 2013; accepted 25 August 2013

Copyright (c) 2013 Gustavo W. Kuster et al. This is an open access article distributed under the Creative Commons Attribution License, which permits unrestricted use, distribution, and reproduction in any medium, provided the original work is properly cited.

\begin{abstract}
The degree of vessel lumen narrowing is an independent predictor of ischemic stroke. New developments in carotid plaque morphology imaging (MR, CT), may bring new insights to the relationship between carotid atherosclerotic disease and stroke risk. Our aim is to review the stroke risk in a symptomatic patient with moderate carotid stenosis by $C T$ imaging and histopathology. A 72-year-old patient with low ABCD2 scores TIA and moderate left internal carotid stenosis ( $50 \%$ by carotid ultrasound), was discharged with an optimized medical therapy. Four months later, he presented an ischemic stroke in the left frontal area. Carotid angiography showed a $60 \%$ stenosis in the leftinternal carotid artery with a regular surface. CT plaque imaging detected a thin fibrous cap with calcification and an intraplaque hemorrhage (highrisk plaque). These findings were confirmed in the histolopathological study of the atherosclerotic plaque performed after the endarterectomy. After 1 year of follow-up, the patient returned independently to his daily activities. We propose, in this study, the inclusion of noninvasive plaque imaging in the evaluation of acute TIA with moderate carotid stenosis to better select patients with higher risk of stroke recurrence.
\end{abstract}

Keywords: Carotid Atherosclerotic Plaque; CT; Plaque Classification

\section{INTRODUCTION}

Carotid atherosclerotic plaque has been identified since our ancient ancestors and some in the recent modern era have been evaluated to the prevention of catastrophic stroke [1]. The report from North America Symptomatic Carotid Endarterectomy Trial (NASCET) in 1991 has stimulated new interest in carotid stenosis and confirmed angiography as a gold standard method to stratify symptomatic patients to endarterectomy [2].

The degree of vessel lumen narrowing is an independent predictor of ischemic stroke, particularly in symptomatic patients with severe carotid stenosis (70\%). Carotid endarterectomy is also associated with a moderate stroke risk reduction in patients with symptomatic moderate carotid stenosis (50\% - 69\%). However, treatment decisions in these cases should be also considered as other important issues including an exceptional surgical skill [3]. In addition, optimal medical treatment has been improved and becomes a topic of equal importance for managing carotid disease, especially in those patients with asymptomatic atherosclerotic plaque [4].

New developments in carotid plaque morphology imaging, particularly MR or CT, may bring new insights to the relationship between carotid atherosclerotic disease and stroke risk [5,6].

Our aim is to review the stroke risk in a symptomatic patient with moderate carotid stenosis according to the plaque surface morphology and the degree of stenosis on carotid angiography and to compare the carotid plaque morphology classification obtained by CT imaging and histopathology.

\section{CASE}

A 72-year-old previously hypertensive patient arrived at emergency department with a sudden onset of right sided weakness lasting 10 minutes. He was evaluated using the TIA assessment protocol and obtained a low 
ABCD2 score. The only remarkable finding was a proximal moderate left internal carotid stenosis (50\%) detected by the carotid ultrasound examination. He was discharged and referred to the neurological outpatient clinic with an optimized medical therapy.

Four mouth later, he presented a recurrence of similar symptoms without a complete recovery (NIHSS $=2$ ) and arrived at hospital outside of the therapeutic window for reperfusion. Brain MRI demonstrated an ischemic stroke lesion in the corona radiata and frontal cortex visible in the FLAIR and T2-weighted imaging. Carotid angiography showed a $60 \%$ stenosis in the left proximal internal carotid artery with a regular surface (Figure 1(A)). CT plaque imaging (Figure 1(B)) detected a thin fibrous cap with calcification and an intraplaque hemorrhage (Figure 1(C)) classified as a high-risk plaque according to the American Heart Association plaque classification [7]. These findings were confirmed in the histolopathological study of the atherosclerotic plaque (Figure 1(D)) performed after the endarterectomy. After 1 year of follow-up, the patient returned independently to his daily activities (modified Rankin score $=1$ ).

\section{DISCUSSION}

Carotid ultrasound is usually the first line examination to evaluate carotid disease in patients with TIA and

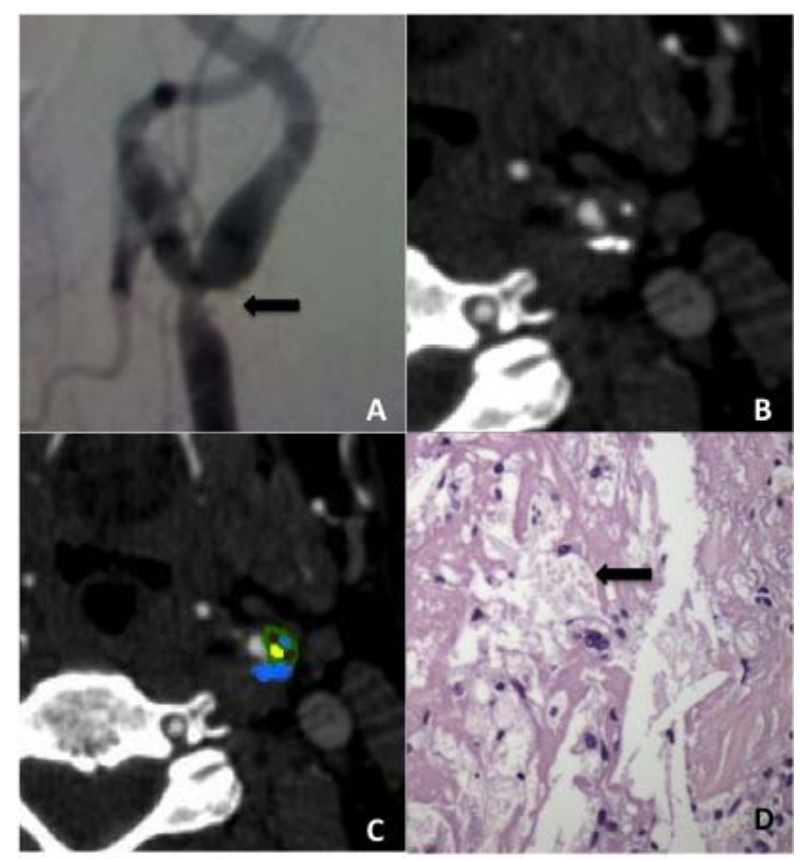

Figure 1. (A) Digital Subtraction Angiography with 60\% bulb carotid stenosis, according NASCET criteria, and regular surface (arrow). (B,C) Contrast CT carotid plaque imaging with a thin fibrous cap (green), calcification (blue), rich lipid necrotic core (yellow) and intraplaque hemorrhage (red). (D) Histological sample (HE, $\times 400)$ of pos endarterectomy carotid plaque confirming hemorrhage (arrow). detects a stenosis degree in the lower limit of the range (50\%) for a clinical decision in favor of carotid endarterectomy in our patients.

Carotid angiography performed in the recurrent ischemic event did not significantly add new information. We hypothesized that the stenosis grade obtained (60\%) might have changed during the interval between the two ischemic events due to dynamic modification in the structure and have turned into a high risk plaque. CT plaque imaging identified features beyond luminal stenosis or plaque surface and represented a new noninvasive imaging technique that might reliably assess plaque vulnerability in symptomatic carotid disease patients presenting with an acute ischemic event. Based on histological American Heart Association criteria, the classification allows categorization of carotid plaques noninvasively into distinct lesion types (I-VIII). Atherosclerotic plaques that are prone to rupture owing to their intrinsic composition such as a large lipid core, thin fibrous cap and intraplaque hemorrhage are associated with subsequent thromboembolic ischemic events as occurred in our patients.

CT plaque imaging classification worked less well for classifying lipid-rich necrotic cores and hemorrhage, probably because the range of densities associated with these components overlapped with the densities associated with connective tissue, but they showed a good correlation with histological classification when only large lipid core and large hemorrhage are considered [5]. On the other hand, MRI has also some limitations in the acute stroke evaluation and needs a specific phased-array surface coil for plaque examination [6].

Timing of carotid endarterectomy after an ischemic event may largely influence outcome. Therefore, we propose the inclusion of noninvasive CT plaque imaging in the evaluation of acute TIA with moderate carotid stenosis to better select patients with higher risk of stroke recurrence.

\section{REFERENCES}

[1] Allam, A.H., Thompson, R.C., Wann, L.S., et al. (2011) Atherosclerosis in ancient Egyptian mummies; the Horus study. JACC Cardiovasc Imaging, 4, 315-327. http://dx.doi.org/10.1016/j.jcmg.2011.02.002

[2] North American Symptomatic Carotid Endarterectomy Trial Collaborators (1991) Beneficial effect of carotid endarterectomy in symptomatic patients with high grade carotid stenosis. The New England Journal of Medicine, 325, 445-453.

http://dx.doi.org/10.1056/NEJM199108153250701

[3] Barnett, J.J.M., Taylor, D.W., Eliasziw, M. et al. (1998) Benefit of carotid endarterectomy in patients with symptomatic moderate or severe stenosis. The New England Journal of Medicine, 339, 1415-1425. 
http://dx.doi.org/10.1056/NEJM199811123392002

[4] Marquadt, L., Geraghty, O.C., Mehta, Z. and Rothwell, P.M. (2010) Low risk of ipsilateral stroke in patients with asymptomatic carotid stenosis on best medical treatment: A prospective, population-based study. Stroke, 41, e11e17. http://dx.doi.org/10.1161/STROKEAHA.109.561837

[5] Wintermark, M., Jawadi, S.S., Rapp, J.H., et al. (2008) High-resolution CT imagin of carotid artery atherosclerotic plaques. American Journal of Neuroradiology, 29, 875-882. http://dx.doi.org/10.3174/ajnr.A0950
[6] Saam, T., Cai, J., Ma, L., Cai, Y.Q., et al. (2006) Comparison of symptomatic and asymptomatic atherosclerotic plaques features with in vivo MR imaging. Radiology, 20, 464-472. http://dx.doi.org/10.1148/radiol.2402050390

[7] Stary, H.C., Chandler, A.B. and Dinsmore, R.E., American Heart Association (1995) A definition types of atherosclerotic lesions and a histological classification of atherosclerosis: A report from the Committee on vascular lesions of the council on atherosclerosis. Circulation, 92, 1355-1374. http://dx.doi.org/10.1161/01.CIR.92.5.1355 